\title{
Rétinoblastome : de l'épidémiologie génétique à la biologie moléculaire
}

A partir d'une étude épidémiologique et d'une analyse statistique classiques, Knudson proposait, en 1971, l'hypothèse selon laquelle le rétinoblastome était la conséquence de deux événements mutationnels, le premier germinal ou somatique, le second toujours somatique. Les données de cytogénétique et de biologie moléculaires devaient, plus de dix ans après, confirmer pleinement ce modèle. Elles devaient aussi démontrer l'origine paternelle préférentielle de la première mutation. En revanche, les mécanismes responsables des différences de pénétrance et d'expressivité observées selon les familles restent incertains. Ces mécanismes pourraient d'ailleurs être divers, impliquant parfois une insertion équilibrée intéressant la bande 13q14 (siège du gène de susceptibilité $\mathbf{R b}$ ), d'autres fois la nature des altérations du locus $\mathbf{R b}$ et des loci environnants, $d$ 'autres fois enfin un fond "génétique " modulant la susceptibilé aux mutations somatiques et (ou) au cancer.

Catherine Bonaïti-Pellié Josué Feingold

\section{ADRESSE}

C. Bonaïti-Pellié : directeur de recherches Inserm. J. Feingold : directeur de recherches Inserm, directeur de l'unité de recherches d'épidémiologie génétique. Unité de recherches d'épidémiologie génétique (Inserm U. 155), Château de Longchamp, Carrefour de Longchamp, Bois

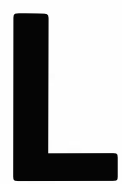

e rétinoblastome est une tumeur embryonnaire de l'œil atteignant un enfant environ sur 20000 , le plus souvent avant l'âge de quatre ans. L'existence de cas familiaux est connue depuis longtemps : un certain nombre d'études ont été réalisées dans les années 1950 à 1970 et ont permis de conclure à l'existence de deux types de rétinoblastome, le type héréditaire et le type non héréditaire (ou sporadique). Une bonne revue de la question a été écrite par Vogel en 1979 [1].

\section{L'hypothèse des deux mutations}

Le type sporadique de rétinoblastome est toujours unilatéral alors que le type héréditaire est plus souvent bilatéral qu'unilatéral et se transmet de façon dominante avec une forte pénétrance. Tous les cas bilatéraux et quelques cas unilatéraux sont donc porteurs d'un gène muté qui leur a été transmis par un parent porteur (atteint ou non) ou bien est le résultat d'une mutation germinale de novo. Un modèle unificateur a été proposé par Knudson en 1971 [2], selon lequel deux événements mutationnels seulement seraient nécessaires à l'apparition d'une tumeur (figure 1). La première mutation serait soit germinale (dans le type héréditaire), soit somatique dans les cellules rétiniennes (dans le type sporadique). Quand la première mutation est germinale, toutes les cellules, en particulier les cellules rétiniennes, sont porteuses de la mutation. La deuxième mutation serait toujours somatique et aboutirait à la formation d'une tumeur dans chaque cellule déjà porteuse de la première mutation. L'apparition de deux mutations somatiques dans la même cellule rétinienne est un événement très rare. Il est donc quasi 
impossible qu'il se produise dans les deux yeux, si bien que le type sporadique ne peut pas être bilatéral. Knudson a utilisé deux sortes de données pour tester son hypothèse. D'une part, il a étudié la proportion de porteurs sains, de cas unilatéraux et de cas bilatéraux parmi les porteurs de la mutation germinale. Si un seul événement mutationnel (rare) est nécessaire pour qu'une cellule donne naissance à une tumeur, compte tenu du très grand nombre de cellules à risque, on s'attend que le nombre de tumeurs suive une loi de Poisson*. Il a calculé les proportions attendues des trois phénotypes (bilatéraux, unilatéraux, porteurs sains) pour différentes valeurs du paramètre $m$ de la loi de Poisson (Tableau I, p. 975). En comparant ces proportions théoriques à celles qui avaient été observées par différents auteurs (bilatéraux : de 60 à $75 \%$, unilatéraux de : 25 à $40 \%$, porteurs sains : de 1 à $10 \%$ ), il a trouvé que les données étaient compatibles avec $m=3$, c'est-à-dire qu'un porteur de la mutation germinale développerait en moyenne trois tumeurs indépendantes pour l'ensemble des deux yeux. D'autre part, il a étudié la décroissance du logarithme du pourcentage de cas non encore diagnostiqués en fonction de l'âge. Il a trouvé une décroissance linéaire, compatible avec l'apparition d'un seul événement (one-hit) dans les cas bilatéraux, et une décroissance parabolique, en accord avec l'apparition de deux événements (two-hit) dans les cas unilatéraux isolés.

L'approche moléculaire a permis d'étayer cette hypothèse et de préciser le locus impliqué dans la maladie.

\section{Le gène du rétinoblastome}

L'observation de quelques cas de rétinoblastome associés à une délétion constitutionnelle de la région $13 q 14$ a fait penser que le premier événement pourrait bien être une mutation

\footnotetext{
- La loi de Poisson donne la probabilité qu'un événement se produise $\mathbf{n}$ fois au sein d'une population de très grand effectif $N$, lorsque la probabilité $\mathrm{p}$ de survenue de cet événement pour un élément donné de cette population est extrêmement petile $P(\mathrm{n})=e^{-\mathrm{m}} \mathrm{m}^{\mathrm{n}} / \mathrm{n}$ ! où $\mathrm{m}$ est le paramètre de la loi de Poisson, c'est-à-dire le nombre mo yen de survenue de l'événement au sein de la population, soit $N$ p. $\mathrm{m} / \mathrm{s} n^{\circ} 10$, vol. 6 , décembre 90
}

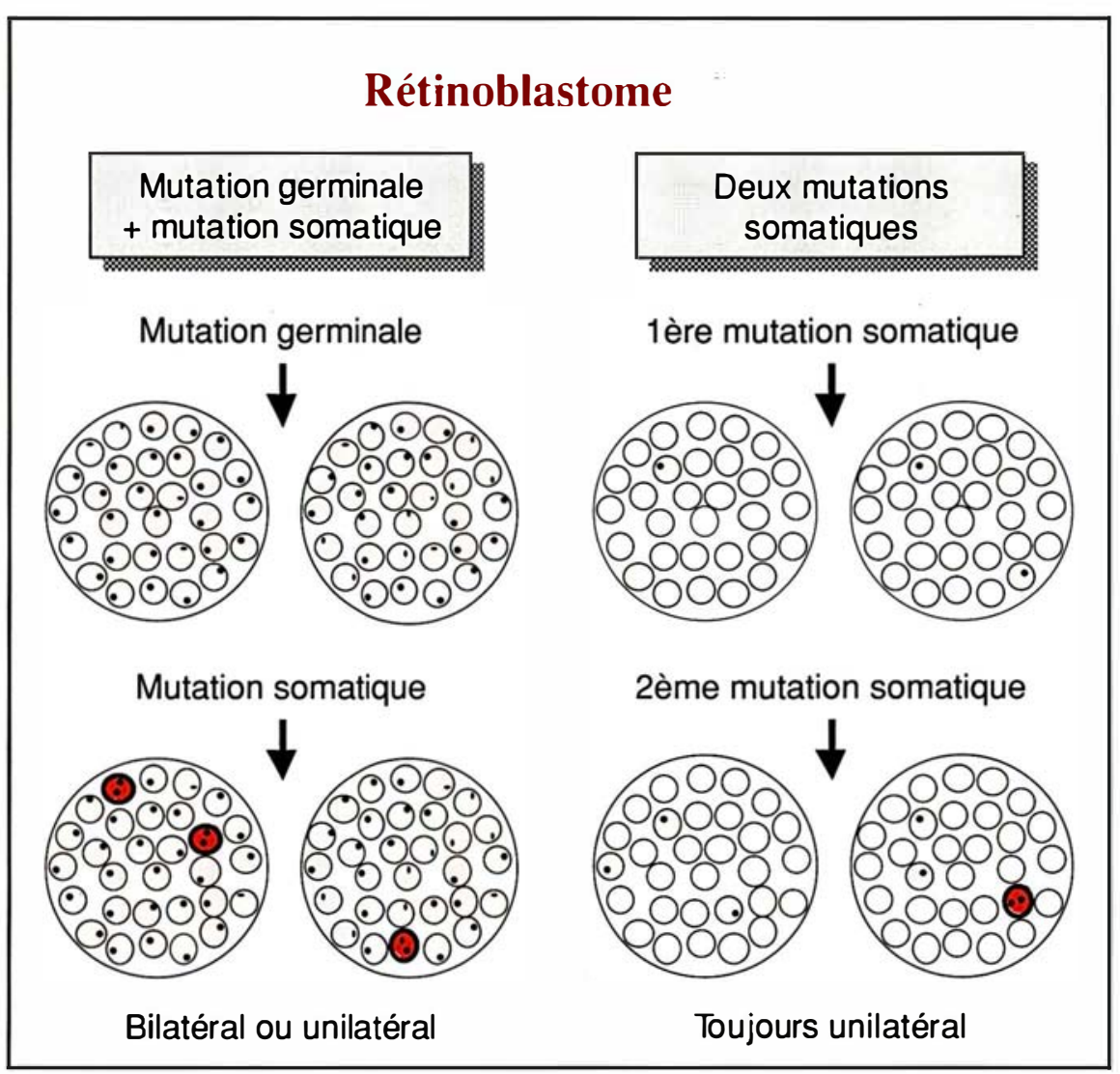

Figure 1. Hypothèse des deux mutations proposée par Knudson en 1971.

à un locus situé dans cette région. Cela a été effectivement montré par Sparkes et al. [3] qui ont trouvé une liaison génétique très étroite entre le rétinoblastome et l'estérase $\mathrm{D}$ dont le locus est justement en 13q14. Le locus fut alors appelé Rb1.

La nature du deuxième événement a été élucidée par des études de marqueurs génétiques, tels que l'estérase $\mathrm{D}[4]$, et des polymorphismes de fragments de restriction [5,6], sur le chromosome 13. En comparant le génotype constitutionnel de patients à celui de leurs tumeurs, ils ont pu montrer que le deuxième événement était une perte fonctionnelle de l'allèle normal sur le locus Rb1 homologue dans une cellule rétinienne. Il s'avérait donc que le rétinoblastome était la conséquence d'une perte totale de gène normal à ce locus et que le caractère tumoral était récessif au niveau cellulaire. C'était la première découverte d'un gène sup- presseur de cancer qui fut appelé antioncogène, par opposition au rôle activateur des oncogènes [7]. Quelques années plus tard, le gène fut cloné et caractérisé par trois groupes [8-10].

\section{Origine parentale de la mutation germinale}

La différence entre le développement des cellules germinales de l'homme et de la femme peut expliquer que la fréquence des mutations ne soit pas identique dans les deux sexes. Les gamètes femelles sont formés après un très petit nombre de divisions cellulaires, de l'ordre de 23 divisions cellulaires chez la femme, alors que chez l'homme plusieurs centaines de divisions sont nécessaires [11]. Si les mutations germinales surviennent au moment de la réplication de l'ADN, on doit s'attendre à un taux de mutation plus élevé chez l'homme 


\section{RÉFÉRENCES}

1. Vogel F. Genetics of retinoblastoma. Hum Genet 197 ; 52 : 1-54.

2. Knudson AG. Mutation and cancer : statistical study of retinoblastoma. Proc Natl Acad Sci USA 1971; 68 : 820-3.

3. Sparkes RS, Murphree AL, Lingua $\mathrm{RW}$, et al. Gene for hereditary retinoblastoma assigned to human chromosome 13 by linkage analysis of esterase D. Science 1983 ; 219: 971-3.

4. Godbout R, Dryja TP, Squirre J, Gallie BL, Philipps RA. Somatic inactivation of genes on chromosome 13 is a common event in retinoblastoma. Nature 1983 ; 304 : 451-3.

5. Cavenee WK, Dryja TP, Phillips RA, et al. Expression of recessive alleles by chromosomal mechanisms in retinoblastoma. Nature 1983 ; 305 : 779-84.

6. Dryja TP, Cavenee $\mathrm{W}$, White $\mathrm{R}$, et al. Homozygosity of chromosome 13 in retinoblastoma. $N$ Engl J Med 1984 ; 310 : 550-3.

7. Knudson AG. Model hereditary cancers of man. Prog Nucleic Acids Res Mol Biol $1983 ; 29: 17-25$.

8. Friend SH, Bernards R, Rogelji S, et al. A human DNA segment with properties of the gene that predisposes to retinoblastoma and osteosarcoma. Nature 1986 ; 323 : 643-6.

9. Fung YKT, Murphree AL, T'Ang A, Qian J, Hinrichs SH, Benedict WF. Structural evidence for the authenticity of the human retinoblastoma gene. Science 1987 ; 236 : 1657-61.

10. Lee WH, Bookstein R, Hong F, Young LH, Shew JY, Lee EYHP. Human retinoblastoma susceptibility gene : cloning, identification and sequence. Science 1987 ; 235 : 1394-9.

11. Vogel F, Rathenberg R. Spontaneous mutation in man. Adv Hum Genet 1975 ; 5 : 223-318.

12. Pellié C, Briard ML, Feingold J, Frézal J. Parental age in retinoblastoma. que chez la femme. Les gènes mutants pouvant s'accumuler avec le temps dans la lignée germinale, on s'attend également à ce que le taux de mutation puisse augmenter avec l'âge. Il y a 17 ans, nous avons recherché si, selon ces critères, les cas bilatéraux isolés de rétinoblastome pouvaient être dus à une mutation récente [12]. Nous avons effectivement trouvé une augmentation de l'âge paternel (et non de l'âge maternel) dans les cas bilatéraux isolés et non dans les cas unilatéraux, suggérant que la forme bilatérale isolée était bien due à une mutation germinale de novo plus fréquente chez l'homme que chez la femme.

Dryja et al. [13] et Zhu et al. [14] ont récemment confirmé ce résultat par des techniques de biologie moléculaire visant à identifier l'origine parentale de la mutation chez respectivement 10 et 5 patients atteints de rétinoblastome bilatéral et qui étaient les premiers cas apparus dans leur famille. Parmi ces 15 mutations de novo, 14 (respectivement 10 et 4 ) se sont produites sur le chromosome 13 venant du père, montrant un excès significatif de mutations d'origine paternelle par rapport à celles d'origine maternelle $(p=0,001)$. Parmi les deux hypothèses proposées par Zhu et al. [14], mutation plus fréquente au cours de la spermatogenèse que de l'ovogenèse, ou bien rôle possible d'une empreinte parentale rendant le gène paternel plus susceptible aux mutations au cours de l'embryogenèse précoce, l'existence d'un âge paternel élevé plaide nettement plus en faveur de la première que de la deuxième.

Le même excès de mutations récentes d'origine paternelle a été trouvé pour des délétions caryotypiquement décelables par Ejima et al. [15] : dans 8 des 9 cas informatifs, le chromosome 13 anormal venait du père $(p=0,04)$.

\section{Pénétrance et expressivité}

C'est Neel [16] qui a noté le premier l'existence de familles comportant des cas de rétinoblastome ayant un lien de parenté éloigné (degré 2 ou plus). Dans notre étude, ces familles comportant au moins deux porteurs sains représentent plus d'un quart de l'ensemble des familles [17]. Cette observation est en désaccord avec la pénétrance quasi complète estimée à partir de la descendance des sujets bilatéraux, et qui implique une très

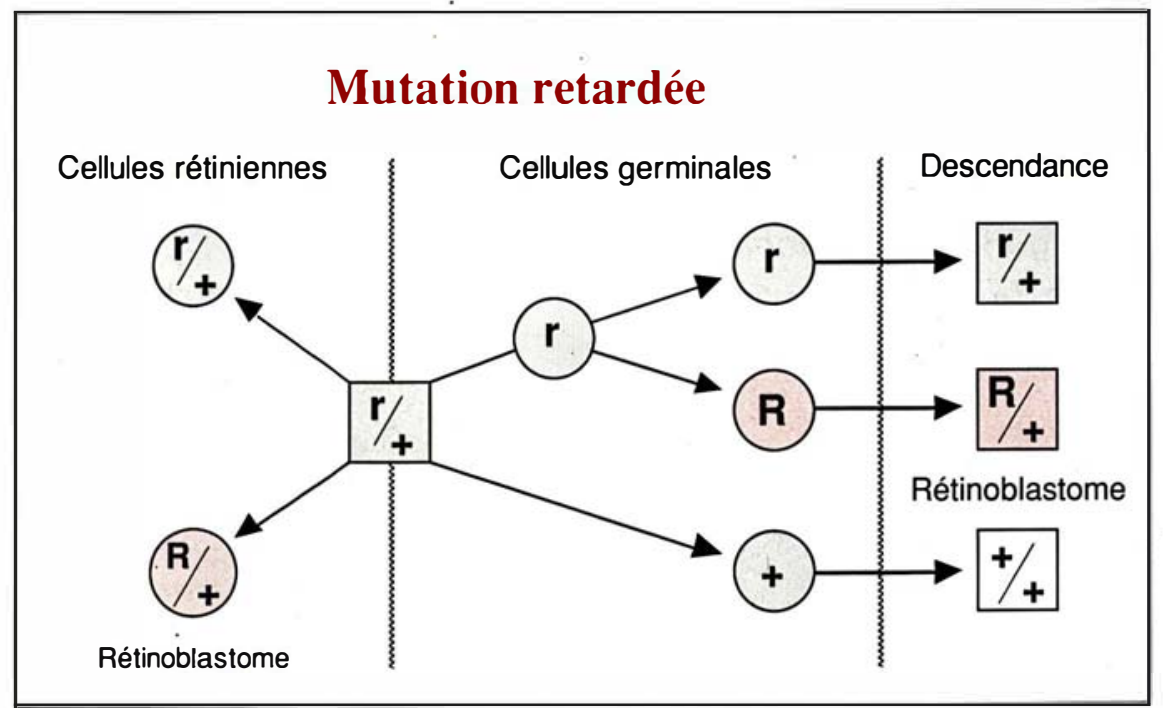

Figure 2. Hypothèse de la mutation retardée proposée par Neel en 1962 et reprise par Hermann en 1976. Les carrés représentent des individus et les ronds des cellules ( $r$ : prémutation, $R$ : mutation complète). 
Tableau I

DISTRIBUTION ATTENDUE DES TUMEURS CHEZ UN PORTEUR DE LA MUTATION GERMINALE

\begin{tabular}{|c|c|c|c|c|c|c|c|c|}
\hline \multirow{2}{*}{$\begin{array}{c}\text { Nombre } \\
\text { total } n \text { de } \\
\text { tumeurs } \\
\text { (deux yeux) }\end{array}$} & \multirow{2}{*}{$\begin{array}{c}\text { Probabilité } \\
\text { (Poisson) }\end{array}$} & \multirow{2}{*}{$\begin{array}{c}\text { Proportion } \\
\text { de cas } \\
\text { unilatéraux } \\
2(1 / 2)^{n}\end{array}$} & \multicolumn{5}{|c|}{ Fréquences attendues pour différentes valeurs de $\mathrm{m}$} & \multirow{2}{*}{$\begin{array}{c}\text { Fréquences } \\
\text { observées }\end{array}$} \\
\hline & & & 1 & 2 & 3 & 4 & 5 & \\
\hline 0 & $e-m$ & $\ldots$ & 0,368 & 0,135 & 0,050 & 0,018 & 0,007 & \\
\hline 1 & me-m & 1 & $0,368 / 0^{*}$ & $0,271 / 0$ & $0,150 / 0$ & $0,073 / 0$ & $0,034 / 0$ & \\
\hline 2 & $\frac{m^{2} e^{-m}}{2 !}$ & $1 / 2$ & $0,092 / 0,092$ & $0,135 / 0,135$ & $0,112 / 0,112$ & $0,073 / 0,073$ & $0,042 / 0,042$ & \\
\hline 3 & $\frac{m^{3} e^{-m}}{3 !}$ & $1 / 4$ & $0,015 / 0,046$ & $0,045 / 0,135$ & $0,056 / 0,168$ & $0,019 / 0,147$ & $0,034 / 0,131$ & \\
\hline 4 & $\frac{m^{4} e^{-m}}{4 !}$ & $1 / 8$ & $0,002 / 0,013$ & $0,011 / 0,079$ & $0,021 / 0,147$ & $0,024 / 0,172$ & $0,021 / 0,148$ & \\
\hline 5 & $\frac{m^{5} e^{-m}}{5 !}$ & $1 / 16$ & $0,000 / 0,003$ & $0,002 / 0,034$ & $0,006 / 0,095$ & $0,010 / 0,147$ & $0,011 / 0,158$ & \\
\hline 6 & $\frac{m^{6} e^{-m}}{6 !}$ & $1 / 32$ & $0,000 / 0,001$ & $0,000 / 0,012$ & $0,002 / 0,049$ & $0,003 / 0,101$ & $0,004 / 0,136$ & \\
\hline 7 & $\frac{m^{7} e^{-m}}{7 !}$ & $1 / 64$ & $\ldots$ & $0,000 / 0,003$ & $0,000 / 0,022$ & $0,001 / 0,059$ & $0,002 / 0,098$ & \\
\hline$\geqslant 8$ & $\sum_{n=8}^{n=\infty} \frac{m^{7} e^{-m}}{7 !}$ & $2(1 / 2)^{n}$ & $\cdots$ & $0,000 / 0,001$ & $0,000 / 0,010$ & $0,000 / 0,050$ & $0,000 / 0,132$ & \\
\hline \multicolumn{3}{|c|}{ Total } & $\begin{array}{l}0,368 \\
0,477 \\
0,155\end{array}$ & $\begin{array}{l}0,135 \\
0,464 \\
0,399 \\
\end{array}$ & $\begin{array}{l}0,050 \\
0,347 \\
0,603 \\
\end{array}$ & $\begin{array}{l}0,018 \\
0,233 \\
0,749\end{array}$ & $\begin{array}{l}0,007 \\
0,148 \\
0,845\end{array}$ & $\begin{array}{l}0,01-0,10 \\
0,25-0,40 \\
0,60-0,75\end{array}$ \\
\hline
\end{tabular}

* Unilatéral/bilatéral

faible probabilité qu'aucune cellule rétinienne d'un porteur du gène ne soit touchée par une mutation somatique. Neel [16] avait suggéré que ces familles pourraient être dues au phénomène de mutation retardée, hypothèse qui avait été proposée la première fois par Auerbach [18] pour expliquer les observations familiales dans un type de malformation de la main. Une prémutation pouvait être transmise sur une ou plusieurs générations et, à un certain moment, transformée en mutation complète (modification que Hermann [19] appelle la télomutation), soit dans les cellules rétiniennes d'un individu, qui pourrait alors développer un rétinoblastome, soit dans les cellules. germinales, le risque de rétinoblastome étant alors pour sa descendance (figure 2).

Par ailleurs, Ellsworth [20] et Matsunaga [21] signalaient que le risque $\mathrm{m} / \mathrm{s} n^{\circ} 10$, vol. 6, décembre 90 pour un enfant pouvait être différent selon l'expressivité du parent porteur du gène : les enfants d'un individu présentant la forme la plus sévère (cas bilatéraux) auraient un risque plus élevé d'être atteints (pénétrance plus élevée) et, dans ce cas, de développer un rétinoblastome bilatéral (degré d'expressivité plus fort) que les enfants des sujets ayant une forme moins sévère (cas unilatéraux). Les enfants des porteurs sains, qui ont le plus faible degré d'expressivité, auraient la pénétrance et l'expressivité les plus faibles. Hermann [19, 22] défendait l'hypothèse que le phénomène de mutation retardée pouvait fort bien expliquer ces différences. En revanche, selon Matsunaga [23], ces différences seraient dues à des degrés différents de "résistance de l'hôte ": un individu ayant le gène du rétinoblastome serait plus ou moins susceptible de développer une tumeur, et l'apparition d'une deuxième mutation ne serait pas nécessaire. Cette susceptibilité serait en partie génétiquement déterminée selon un mode polygénique avec deux seuils divisant la population des porteurs du gène en trois catégories correspondant aux trois phénotypes possibles : les porteurs sains, les unilatéraux et les bilatéraux.

Nous avons étudié ces hypothèses [24] à partir de 166 généalogies de rétinoblastomes supposés héréditaires appartenant à neuf études de la littérature y compris la nôtre [17, 20, 25-30], en utilisant la méthode du maximum de vraisemblance pour les tests d'hypothèses et les estimations de paramètres.

Dans un premier temps, nous avons pu confirmer que, selon le phénotype des parents porteurs de rétinoblastomes bilatéraux, unilatéraux ou porteurs sains, le risque chez l'enfant 


\section{RÉFÉRENCES}

13. Dryja TP, Mukai S, Petersen R, Rapaport JM, Walton D, Yandell DW. Parental origin of mutations of the retinoblastoma gene. Nature 1989 ; 339 : 556-8.

14. Zhu X, Dunn JM, Philips RA, et al. Prefential germline mutation of the paternal allele in retinoblastoma. Nature 1989 ; $340: 312-3$.

15. Ejima Y, Sasaki MS, Kaneko A, Tanooka H. Types, rates, origin and expressivity of mutations involving $13 \mathrm{q} 14$ in retinoblastoma patients. Hum Genet 1988 ; $79: 118-23$.

16. Neel JV. Mutations in the human population. In : Burdette WJ, ed. Methodology in Human Genetics. San Francisco : Holden Day, 1962 ; 203-79.

17. Briard-Guillemot ML, Bonaïti-Pellié C, Feingold J, Frézal J. Etude génétique du rétinoblastome. Humangenetik 1974; 24 : 271-84.

18. Auerbach C. A possible case of delayed mutation in man. Ann Hum Genet 1956 ; $20: 266-9$.

19. Hermann J. Delayed mutation model : carotid body tumors and retinoblastoma. In : Mulvihill JJ, Miller RW, Fraumeni JF, eds. Genetics of Human Cancer. New York : Raven Press, 1977 ; 417-38.

20. Ellsworth RM. The practical management of retinoblastoma. Trans Am Ophthalmol Soc 1969 ; 67 : 462-534.

21. Matsunaga E. Hereditary retinoblastoma : penetrance, expressivity and age at onset. Hum Genet $1976 ; 33: 1-15$.

22. Hermann J. Delayed mutation as a cause of retinoblastoma: application to genetic counseling. Birth Defects $1976 ; 12$ : 79-90.

23. Matsunaga E. Hereditary retinoblastoma : delayed mutation or host resistance? Am J Hum Genet 1978 ; 30 : 406-24.

24. Bonaïti-Pellié C, Briard-Guillemot ML. Segregation analysis in hereditary retinoblas-

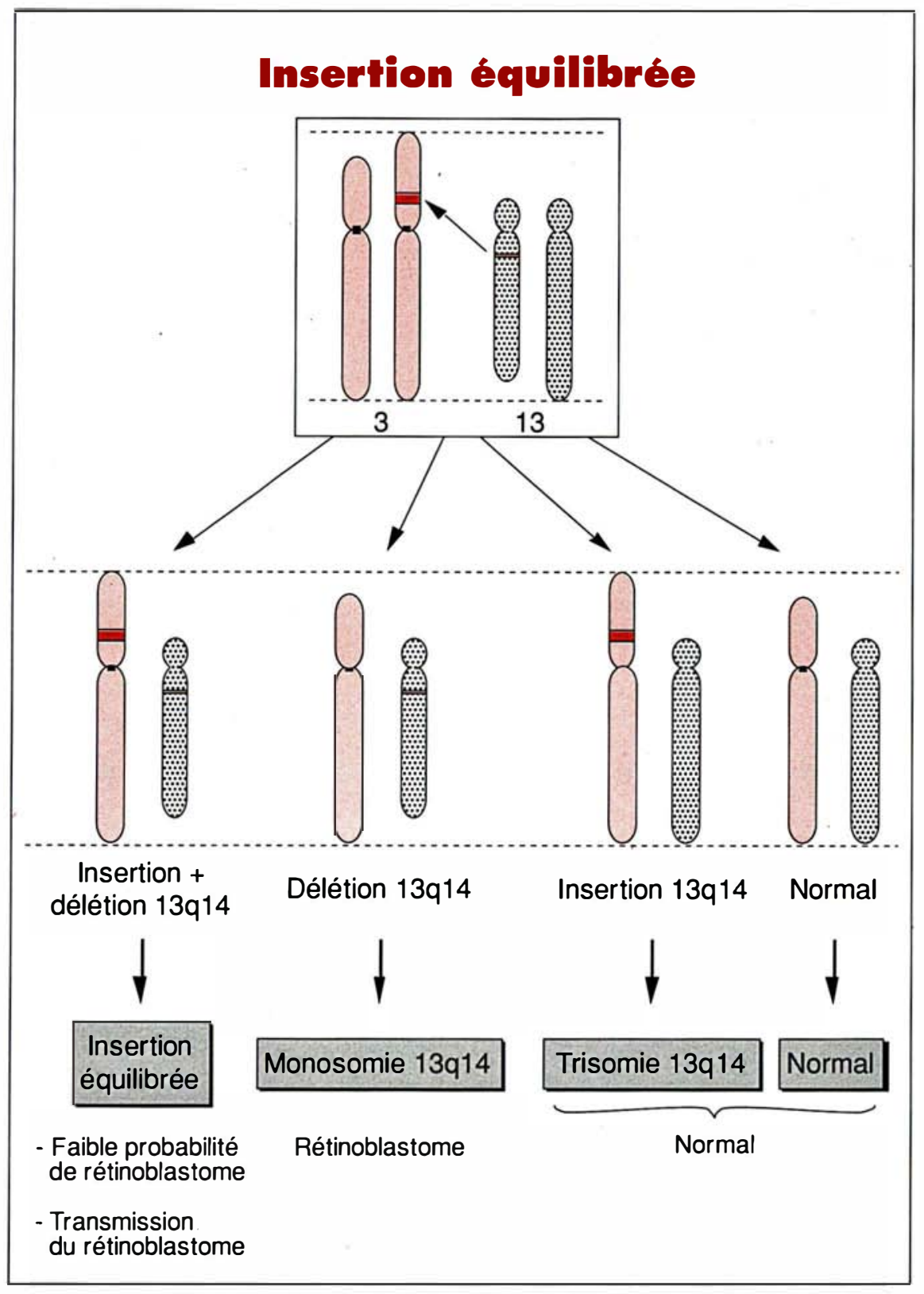

Figure 3. Hypothèse de I'insertion équilibrée. Ici, un fragment $13 q 14$ est inséré dans le bras court du chromosome 3 (exemple de Strong et al., 1981).

décroît. En outre, on trouve une plus forte proportion de rétinoblastomes bilatéraux parmi les enfants atteints lorsque le parent porteur est bilatéral $(0,87)$ que lorsqu'il est unilatéral $(0,76)$ ou porteur sain $(0,60)$. En outre, même en ayant très soigneusement tenu compte du mode de recensement des familles, nous avons trouvé que les enfants des unilatéraux et des porteurs sains ont un risque significativement plus élevé lorsqu'ils ont déjà un germain atteint.

Dans un deuxième temps, nous avons supposé l'existence de deux types de transmetteurs du gène parmi les unilatéraux et les porteurs sains : les transmetteurs faibles (porteurs 
d'une prémutation) et les transmetteurs forts (porteurs d'une mutation complète). Nous avons trouvé que parmi les transmetteurs forts, il y avait toujours une différence de risque entre les enfants des cas bilatéraux $(0,49)$ et ceux des unilatéraux et des porteurs sains $(0,31)$, apportant des arguments contre l'hypothèse selon laquelle la mutation retardée pourrait entièrement expliquer les différences de pénétrance et d'expressivité.

\section{L'hypothèse de l'insertion équilibrée}

Ce sont les cytogénéticiens qui ont fourni une des explications possibles pour la transmission du rétinoblastome par des individus non atteints. Riccardi et al. [32], Rivera et al. [33], Strong et al. [34] et Turleau et al. [35] ont ainsi publié des observations d'insertions équilibrées qui peuvent produire dans la descendance soit des trisomies partielles $13 \mathrm{q} 14$, soit des monomies partielles 13 q14 avec rétinoblastome, soit des insertions équilibrées (figure 3). Ces mécanismes peuvent représenter une explication cytogénétique de l'hypothèse de la mutation retardée, l'insertion équilibrée représentant la prémutation et la délétion $13 q 14$, la mutation complète. A partir des mêmes études de la littérature et en utilisant toujours la méthode du maximum de vraisemblance, nous avons réanalysé les données pour tester si ce mécanisme pouvait expliquer les différences de distribution entre les familles [36].

Les vraisemblances des modèles pour les données familiales observées ont été calculées en supposant trois génotypes : les porteurs de l'insertion équilibrée, les porteurs de la délétion, et le génotype normal (y compris les trisomiques $13 \mathrm{q} 14$ qui ont habituellement un phénotype normal). Trois phénotypes ont été considérés : unilatéraux, bilatéraux et non atteints. Nous avons supposé que tous les bilatéraux étaient porteurs de la délétion (qui pouvait être a minima une mutation ponctuelle) et nous avons

Tableau II

ANALYSE DE SÉGRÉGATION DU RÉTINOBLASTOME HÉRÉDITAIRE SELON LE PHÉNOTYPE DU PARENT TRANSMETTEUR SOUS L'HYPOTHĖSE D'INSERTION ÉQUILIBRÉS 1) EN SUPPOSANT UNE ÉGALE VIABILITÉ DES GAMĖTES

2) EN ADMETTANT LA POSSIBILITÉ D'UNE VIABILITÉ INFÉRIEURE DES GAMĖTES DÉSÉQUILIBRÉS

\begin{tabular}{|c|c|c|c|c|c|}
\hline & \multicolumn{3}{|c|}{ Phénotype du parent transmetteur } & \multirow{2}{*}{\multicolumn{2}{|c|}{$\begin{array}{l}\text { Valeurs attendues } \\
\text { des paramètres sous l'hy- } \\
\text { pothèse d'homogénéité ** }\end{array}$}} \\
\hline & Bilatéral* & Unilatéral & Porteur sain & & \\
\hline $\begin{array}{l}\text { (1) Égale viabilité des gamètes } \\
\text { Probabilité du phénotype chez un por- } \\
\text { teur de la délétion }\left[\begin{array}{l}\mathrm{Bi} \\
\text { Uni } \\
\mathrm{Ps}\end{array}\right. \\
\text { Probabilité du phénotype chez un } \\
\text { porteur de l'insertion équilibrée }\left[\begin{array}{l}\mathrm{Bi} \\
\text { Uni } \\
\mathrm{Ps}\end{array}\right.\end{array}$ & $\begin{array}{l}0,83 \\
0,16 \\
0,01\end{array}$ & $\begin{array}{l}0,54 \\
0,39 \\
0,07\end{array}$ & $\begin{array}{l}0,52 \\
0,40 \\
0,08\end{array}$ & $\begin{array}{c}0,64 \\
0,32 \\
0,04 \\
\\
\\
0 \\
0,12 \\
0,88\end{array}$ & $p<0,01$ \\
\hline $\begin{array}{l}\text { (2) Viabilité inférieure des gamètes } \\
\text { déséquilibrés } \\
\text { Probabilité du phénotype chez un por- } \\
\text { teur de la délétion }\left[\begin{array}{l}\mathrm{Bi} \\
\text { Uni } \\
\mathrm{Ps}\end{array}\right.\end{array}$ & $\begin{array}{l}0,83 \\
0,16 \\
0,01\end{array}$ & $\begin{array}{l}0,62 \\
0,33 \\
0,05\end{array}$ & $\begin{array}{l}0,58 \\
0,36 \\
0,06\end{array}$ & $\begin{array}{l}0,69 \\
0,28 \\
0,03\end{array}$ & \\
\hline $\begin{array}{l}\text { Probabilité du phénotype chez un porteur } \\
\text { de l'insertion équilibrée } \\
{\left[\begin{array}{l}\mathrm{Bi} \\
\text { Uni } \\
\mathrm{Ps}\end{array}\right.}\end{array}$ & $\begin{array}{l}- \\
-\end{array}$ & $\begin{array}{c}0 \\
0,14 \\
0,86\end{array}$ & $\begin{array}{c}0 \\
0,08 \\
0,92\end{array}$ & \begin{tabular}{l|}
0 \\
0,18 \\
0,82
\end{tabular} & $p<0,02$ \\
\hline $\begin{array}{l}\text { Viabilité différentielle } \\
\text { des gamètes }\end{array}\left[\begin{array}{l}\text { avec délétion } \\
\text { avec insertion }\end{array}\right.$ & $\begin{array}{l}1 \\
-\end{array}$ & $\begin{array}{c}0,37 \\
1\end{array}$ & $\begin{array}{c}0,53 \\
1\end{array}$ & $\begin{array}{c}0,57 \\
1\end{array}$ & \\
\hline
\end{tabular}

* Les bilatéraux sont toujours porteurs d'une délétion, ce qui explique l'absence de chiffre correspondant aux insertions dans cette colonne. ** C'est-à-dire absence de différence selon le phénotype du parent transmetteur. 


\section{RÉFÉRENCES}

25. Falls HF, Neel JV. Genetics of retinoblastoma. Arch Ophtalmol 1951 ; 46 : 367-89.

26. Tucker DP, Streinberg AG, Cogan DG. Frequency of genetic transmission of sporadic retinoblastoma. Arch Ophthalmol 1957 ; 57 : 532-5

27. Vogel F. Neue Untersuchungen zur Genetik des Retinoblastoms. Z Mensch Vererb u Konstil Lehre 1957 ; 34 : 205-36.

28. Macklin MT. A study of retinoblastoma in Ohio. Am J Hum Genet $1960 ; 12$ : $1-43$

29. Schappert-Kimmijser J, Hemmes GD, Nijland R. The heredity of retinoblastoma. Ophthalmologica 1966 ; 151 : 197-213.

30. Sorsby A. Bilateral retinoblastoma : a dominantly inherited affection. $\mathrm{Br} \mathrm{Med} J$ 1972 ; 2 : $580-3$

31. Matsunaga E, Ogyu H. Retinoblastoma in Japan : follow up survey of sporadic cases. Jap J Ophthalmol 1976 ; 20 : 266-82.

32. Riccardi VM, Hittner HM, Francke U, et al. Partial triplication and deletion of 13q : Study of a family presenting with bilateral retinoblastomas. Clin Genet $1979 ; 15$ : $332-45$.

33. Rivera H, Turleau C, de Grouchy J, Junien C, Despoisses S, Zücker JM. Retinoblastoma-del (13q14) : report of two patients, one with a trisomic sib due to maternal insertion. Gene dosage effect for esterase D. Hum Genet 1981; 59 : 211-4.

34. Strong LC, Riccardi VM, Ferrell RE Sparkes RS. Familial retinoblastoma and chromosome 13 deletion transmitted via an insertional translocation. Science $1981 ; 213$ : 1501-3.

35. Turleau C, de Grouchy J, ChavinColin F, Despoisses S, Leblanc A. Two cases of del (13q)-retinoblastoma and two cases of partial trisomy due to familial insertion. Ann Genet 1983; $26: 158-60$

36. Bonaïti-Pellié C, Clerget-Darpoux F, Babron MC. Hereditary retinoblastoma can balanced insertion entirely explain the differences of expressivity among families? négligé l'apparition de cas sporadiques dans ces familles.

Pour tester si les différences de distribution de la maladie entre les familles pouvaient être entièrement expliquées par des insertions équilibrées, nous avons effectué des tests d'homogénéité selon le phénotype du parent transmetteur sous deux hypothèses différentes, à savoir une égalité de viabilité de tous les gamètes d'une part, et une viabilité plus faible des gamètes déséquilibrés d'autre part.

Sous l'hypothèse d'une égale viabilité de tous les gamètes, nous avons trouvé une hétérogénéité significative entre familles selon le phénotype du parent transmetteur $\left(\chi^{2}{ }_{4}=13,61\right.$, $p<0,01)$. En laissant varier la probabilité de transmission des gamètes, nous avons trouvé une différence similaire entre familles avec un rejet significatif de l'homogénéité $\left(\chi^{2}{ }_{8}=20,26, p<0,02\right)$. Le Tableau II (p. 977) indique les estimations des différents paramètres selon le phénotype du parent transmetteur sous ces deux hypothèses, ainsi que ces estimations tous phénotypes parentaux confondus. Dans le cas où l'hypothèse d'insertion équilibrée expliquerait les différences d'expressivité entre les familles, on devrait s'attendre à ce que les estimations des paramètres soient identiques quel que soit le phénotype du parent transmetteur et donc égales aux valeurs attendues sous l'hypothèse d'homogénéité, ce qui n'est absolument pas le cas. Cette analyse nous a donc permis de montrer que l'hypothèse de l'insertion équilibrée ne peut expliquer à elle seule les différences de pénétrance et d'expressivité observées dans la descendance des porteurs du gène selon leur phénotype.

\section{Autres hypothèses}

Même si l'existence d'insertions équilibrées a été prouvée comme étant à l'origine de distributions familiales particulières, nous avons vu que ce mécanisme ne pouvait pas expliquer l'ensemble des observations. D'autres hypothèses peuvent être avancées.

La première hypothèse qui vient à l'esprit est que la probabilité de la deuxième mutation, et donc de la formation d'une tumeur chez un porteur du gène, pourrait être génétiquement déterminée, mais dépendante d'autres gènes que la mutation $\mathrm{Rb}$. Cette hypothèse présente quelques ressemblances avec la théorie de la résistance de l'hôte prônée par Matsunaga [23], avec la différence fondamentale que celui-ci ne croyait pas, comme nous l'avons vu, à la nécessité d'un deuxième événement. Nous avions étudié indirectement cette hypothèse en examinant la proportion de décès par cancer chez les grandsparents des enfants atteints de rétinoblastome [37]. Nous avons trouvé un excès de décès par cancer, suggérant l'existence d'un facteur non spécifique de susceptibilité au cancer. Cependant, en étudiant cet excès, d'une part dans les branches paternelle et maternelle des familles, d'autre part selon le type de transmission familiale, nous avons trouvé des arguments en faveur d'une influence de ce facteur sur le taux de la première mutation et non de la deuxième.

La deuxième hypothèse est que la probabilité de formation d'une tumeur pourrait dépendre de la nature de la mutation germinale $\mathrm{D}$. Supposons que la mutation $\mathrm{Di}$ induise une plus grande probabilité de recombinaison somatique ou de ségrégation anormale des chromosomes à la mitose que la mutation $\mathrm{Dj}$, alors la probabilité de formation d'une tumeur devrait être plus grande pour les porteurs de Di que pour les porteurs de Dj. Il se peut aussi, comme Knudson [7] l'avait suggéré, que l'apparition d'un deuxième événement aboutisse à une cellule moins viable si l'individu est porteur de Dj que s'il est porteur de Di. En effet, s'il existe sur la partie délétée du chromosome 13 un allèle létal à l'état homozygote pour la cellule, l'apparition d'une délétion sur le chromosome homologue entraînera la mort de la cellule et aucune tumeur n'apparaîtra. Dryja et al. [6] ont suggéré une explication similaire à l'observation faite par Matsunaga [38] d'une bilatéralité moins fréquente chez les patients ayant une délétion constitutionnelle caryotypiquement décelable que chez ceux où l'on n'observait pas de délétion.

Dans la première hypothèse, la probabilité de formation d'une tumeur dépend du génotype des deux parents et devrait donc varier d'une généra- 
tion à l'autre. Dans la deuxième, quel que soit le mécanisme impliqué (probabilités différentes du deuxième événement, ou viabilités différentes des cellules ayant acquis ce deuxième événement), cette probabilité devrait être identique d'une génération de porteur du gène à l'autre. Les données familiales disponibles à l'heure actuelle ne permettent malheureusement pas de trancher entre ces deux hyputhèses, car il n'est pas possible de tester - dans une même famille - l'égalité de la probabilité individuelle de formation d'une tumeur au cours des générations successives.

La génétique moléculaire pourrait être un précieux apport pour tester ces hypothèses. A notre connaissance, il n'y a qu'une seule étude publiée sur la détection systématique de porteurs sains par l'analyse des marqueurs de l'ADN dans et de part et d'autre du gène $\mathrm{Rb}$ sur le chromosome 13 [39]. Parmi 19 familles de rétinoblastome héréditaire, il y avait 18 familles informatives. Les auteurs ont pu mettre en évidence un porteur sain à la dernière génération dans deux d'entre elles. $\mathrm{La}$ nature de la mutation n'a pu être identifiée que dans une seule famille (autre que les deux précédentes) où il s'agissait d'une délétion du gène entier chez trois membres de la famille, tous atteints.

La biologie moléculaire n'a donc pas pour l'instant d'arguments en faveur d'une de ces hypothèses. Une bonne stratégie d'investigation consisterait à sélectionner fortement deux types de familles : celles comportant des porteurs sains et des cas unilatéraux, d'une part, et celles présentant une transmission régulière de la maladie avec prédominance de ces bilatéraux, d'autre part. Des études systématiques de la nature de la mutation dans ces deux types de familles permettraient probablement de répondre à ces questions importantes et, par la même occasion, de déceler l'existence d'insertions équilibrées non décelables sur le caryotype, mais détectables uniquement au niveau moléculaire, et d'en évaluer la fréquence. Ces études devraient aboutir non seulement à établir des risques plus précis pour une famille donnée, mais surtout à mieux comprendre certains mécanismes à l'origine des altérations de l'ADN

$\mathrm{m} / \mathrm{s} n^{\circ} 10$, vol. 6 , décembre 90

\section{Summary}

Retinoblastoma : from genetic epidemiology to molecular bio$\log y$

The retinoblastoma story is a very good example of mechanisms assessed by genetic epidemiology and confirmed several years later by molecular genetics. The two mutation process proposed by Knudson has been clearly demonstrated by cytogenetic studies and the analysis of genetic markcrs in tumors. Similarly, the excess of new mutations of paternal origin, evidenced by molecular biology techniques, had already been assessed by the analysis of paternal age in bilateral isolated cases. There are still some questions raised by the analysis of family data, such as the differences of penetrance and expressivity among families, which remain unsolved. The balanced insertion hypothcsis, proposed by cytogeneticists, has recently been shown by genetic epidemiologists to be unable to entirely cxplain these differences. There is no doubt that molecular genetic approaches, using an appropriate strategy, will provide the expected answers.

\section{TIRÉS A PART}

C. Bonaïti-Pellié. 